\title{
Modelling Heat and Drought Adaptation in Crops ${ }^{\dagger}$
}

\author{
Erik van Oosterom 1,* , Greg McLean ${ }^{2}$, Kurt Deifel ${ }^{1}$, Vijaya Singh ${ }^{1}$, David Jordan ${ }^{3}$ \\ and Graeme Hammer ${ }^{1}$ \\ 1 Queensland Alliance for Agriculture and Food Innovation (QAAFI), The University of Queensland, \\ Brisbane, QLD 4072, Australia; k.deifel@uq.edu.au (K.D.); v.singh@uq.edu.au (V.S.); \\ g.hammer@uq.edu.au (G.H.) \\ 2 Agri-Science Queensland, Department of Agriculture and Fisheries, Toowoomba, QLD 4350, Australia; \\ g.mclean@uq.edu.au \\ 3 Queensland Alliance for Agriculture and Food Innovation (QAAFI), The University of Queensland, \\ Warwick, QLD 4370, Australia; david.jordan@uq.edu.au \\ * Correspondence: erik.van.oosterom@uq.edu.au \\ + Presented at the third International Tropical Agriculture Conference (TROPAG 2019), Brisbane, Australia, \\ 11-13 November 2019.
}

Published: 8 April 2020

\begin{abstract}
Drought and heat stress are increasingly important abiotic limitations to productivity of sorghum. Here, we use long-term simulations to quantify the importance of transpiration rates to drought adaptation and the importance of threshold temperatures and tolerance above the threshold for adaptation of seed set to heat stress. Simulations were parameterised using results of detailed physiological studies. The importance of transpiration rates to drought adaption was studied by comparing productivity of maize and 3dwarf sorghum. These crops have similar transpiration efficiency but contrasting transpiration rates per unit green leaf area (TGLA), which was captured through differences in RUE. Results showed that the greater TGLA of maize reduced productivity under drought stress, but increased productivity in well-watered conditions, indicating a trade-off between yield potential and drought adaptation. The increased water use of maize associated with higher yield potential could negatively affect carry-over of soil water in a cropping systems context. Simulations for sorghum productivity under heat stress, using long-term weather records for six locations across the Australian sorghum belt, showed that the most common incidence of heat stress around anthesis was the occurrence of individual days with maximum temperatures of $36-38{ }^{\circ} \mathrm{C}$. Because these temperatures were near the threshold that limits seed set, increased temperature thresholds generally minimised yield reductions. However, predicted temperature increases in coming decades justify additional selection for increased tolerance above the threshold. As manipulating sowing dates did not reduce risks of heat stress around anthesis, genetic improvement provides the best prospect to mitigate adverse effects on grain yield.
\end{abstract}

Keywords: maize; radiation use efficiency; seed set; simulation model; sorghum; temperature threshold; transpiration rate 